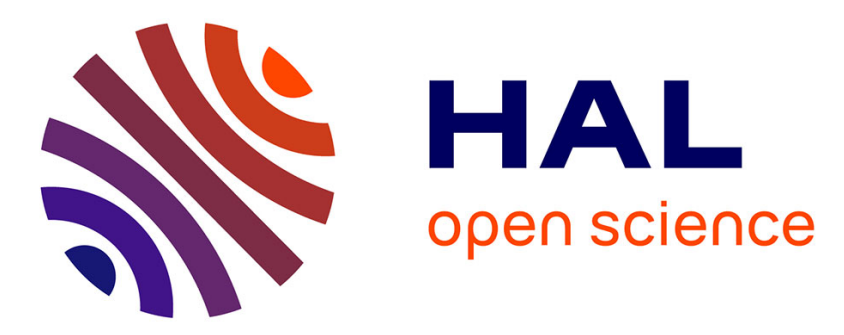

\title{
Ein neuartiger Zugang zu Polyoxomolybdaten mit komplexen Gegenionen - Synthese und Struktur von [Mn(CH3OH)6][Mo8O16(OCH3)8(C2O4)]
}

\author{
Anke Sterzik, Helmar Görls, Wolfgang Imhof
}

\section{- To cite this version:}

Anke Sterzik, Helmar Görls, Wolfgang Imhof. Ein neuartiger Zugang zu Polyoxomolybdaten mit komplexen Gegenionen - Synthese und Struktur von $[\mathrm{Mn}(\mathrm{CH} 3 \mathrm{OH}) 6][\mathrm{Mo} 8 \mathrm{O} 16(\mathrm{OCH} 3) 8(\mathrm{C} 2 \mathrm{O} 4)]$. Journal of Inorganic and General Chemistry / Zeitschrift für anorganische und allgemeine Chemie, 2009, 635 (11), pp.1594. 10.1002/zaac.200801200 . hal-00509228

\section{HAL Id: hal-00509228 https://hal.science/hal-00509228}

Submitted on 11 Aug 2010

HAL is a multi-disciplinary open access archive for the deposit and dissemination of scientific research documents, whether they are published or not. The documents may come from teaching and research institutions in France or abroad, or from public or private research centers.
L'archive ouverte pluridisciplinaire HAL, est destinée au dépôt et à la diffusion de documents scientifiques de niveau recherche, publiés ou non, émanant des établissements d'enseignement et de recherche français ou étrangers, des laboratoires publics ou privés. 


\section{Zeitschrift für Anorganische und}

Allgemeine Chemie

\section{Ein neuartiger Zugang zu Polyoxomolybdaten mit komplexen Gegenionen - Synthese und Struktur von [Mn(CH3OH)6][Mo8016(OCH3)8(C2O4)]}

\begin{tabular}{|r|l|}
\hline Journal: & Zeitschrift für Anorganische und Allgemeine Chemie \\
\hline Wanuscript ID: & zaac-2008-00200.R1 \\
\hline Diley - Manuscript type: & Article \\
\hline Author: & 10-Jun-2009 \\
\hline & $\begin{array}{l}\text { Complete List of Authors: } \\
\text { Anorrs, Helmar; Friedrich-Schiller-Universität, Institut für } \\
\text { Imhof, Wolfgang; Friedrich-Schiller-Universität, Institut für } \\
\text { Anorganische und Analytische Chemie }\end{array}$ \\
\hline Keywords: & $\begin{array}{l}\text { Molybdenum, Manganese, Polyoxometallates, Metal-metal multiple } \\
\text { bonds, X-ray }\end{array}$ \\
\hline
\end{tabular}

\section{s ScholarONE" \\ Manuscript Central}




\title{
Ein neuartiger Zugang zu reduzierten Polyoxomolybdaten mit komplexen Gegenionen - Synthese und Struktur von $\left[\mathrm{Mn}\left(\mathrm{CH}_{3} \mathrm{OH}\right)_{6}\right]\left[\mathrm{Mo}_{8} \mathrm{O}_{16}\left(\mathrm{OCH}_{3}\right)_{8}\left(\mathrm{C}_{2} \mathrm{O}_{4}\right)\right]$
}

\section{A new synthetic route to reduced polyoxomolybdates with complex counter ions - synthesis and structure of $\left[\mathrm{Mn}\left(\mathrm{CH}_{3} \mathrm{OH}\right)_{6}\right]\left[\mathrm{Mo}_{8} \mathrm{O}_{16}\left(\mathrm{OCH}_{3}\right)_{8}\left(\mathrm{C}_{2} \mathrm{O}_{4}\right)\right]$}

Anke Sterzik, Helmar Görls, Eike T. Spielberg, Winfried Plass, Wolfgang Imhof*

Friedrich-Schiller-Universität, Institut für Anorganische und Analytische Chemie, August-Bebel-Str. 2, 07743 Jena / Germany, Fax: +49-3641-948102, E-mail:Wolfgang.Imhof@uni-jena.de

\begin{abstract}
The tetranuclear compound $\left[\mathrm{Mo}_{2}\left(\mathrm{O}_{2} \mathrm{C}-t-\mathrm{Bu}\right)_{3}\right]_{2}\left(\mu-\mathrm{C}_{2} \mathrm{O}_{4}\right), \mathbf{1}$, that is prepared from $\left[\mathrm{Mo}_{2}\left(\mathrm{O}_{2} \mathrm{C}-\mathrm{t}-\mathrm{Bu}\right)_{3}\right]_{4}$ and oxalic acid, was reacted with $\mathrm{MnI}_{2} \times 2 \mathrm{THF}$ to form the polyoxomolybdate compound $\left[\mathrm{Mn}\left(\mathrm{CH}_{3} \mathrm{OH}\right)_{6}\right]\left[\mathrm{Mo}_{8} \mathrm{O}_{16}\left(\mathrm{OCH}_{3}\right)_{8}\left(\mathrm{C}_{2} \mathrm{O}_{4}\right)\right]$, 2, in a complex redox reaction. Crystals of $\mathbf{2}$ were analyzed by single crystal X-ray diffraction showing a octanuclear polyoxomolybdate dianion in which the $\mathrm{Mo}=\mathrm{O}$ moieties are alternately connected via $\mu$-oxo and $\mu$-methoxo units. Charge balance in $\mathbf{2}$ is realized by a manganese(II) cation that is octahedrally coordinated by methanol ligands. The crystal structure is dominated by strong hydrogen bond interactions of the $\mathrm{O}-\mathrm{H}----\mathrm{O}$ type of methanol molecules coordinated to manganese as well as additional methanol molecules in the crystal lattice.
\end{abstract}

Keywords: Molybdenum; Manganese; Polyoxometallates; Metal-metal multiple bonds; X-ray 


\section{Einleitung}

Die Chemie der polynuklearen Oxometallate wird von Vanadium, Wolfram und Molybdän wegen ihrer umfangreichen Anwendungsgebiete, z.B. als Katalysatoren, dominiert. Polyoxometallate zeichnen sich außerdem durch ihre hohe Symmetrie aus. Unterschiede in der Zusammensetzung und Struktur der Polyoxometallat-Cluster führen zu einer Vielzahl an Anwendungsmöglichkeiten. So wurden z.B. cyclische Polyoxometallate unterschiedlicher Ringgröße mit im Hohlraum des Clusters enthaltenen organischen Resten, z.B. Oxalat, synthetisiert und charakterisiert [1a-f].

In der Literatur sind verschiedene gemischtvalente Polyoxometalate aber auch vollständig reduzierte Polyoxomolybdate $(\mathrm{V})$ bereits beschrieben worden [2a-e]. Ausgehend von Oxohalomolybdat $(\mathrm{V})\left[\mathrm{MoOCl}_{4}\left(\mathrm{H}_{2} \mathrm{O}\right)\right]^{-}$und $\left[\mathrm{MoOBr}_{4}\right]^{-}$wurden mit Oxalsäure, einem Alkohol und einem Pyridinderivat R-Py dinukleare Oxomolybdat(V) Komplexe $\left[\mathrm{Mo}_{2} \mathrm{O}_{4}\left(\mu^{2}-\mathrm{C}_{2} \mathrm{O}_{4}\right)_{2}(\mathrm{R}\right.$ $\left.\mathrm{Py})_{2}\right]^{2-}$ erhalten [3]. Ebenso können in Polyoxoanionen andere Atome eingeführt werden, wie zum Beispiel Schwefel. $\left[\mathrm{Mo}_{2} \mathrm{~S}_{2} \mathrm{O}_{2}\right]^{2+}$ Baueinheiten wurden z.B. durch selektive Oxidation der terminalen $\mathrm{S}_{2}$-Liganden des Dianions $\left[\left(\mathrm{S}_{2}\right) \mathrm{MoO}\left(\mu_{2}-\mathrm{S}\right)_{2} \mathrm{MoO}\left(\mathrm{S}_{2}\right)\right]^{2-}$ und durch Zugabe von $\mathrm{KOH}$ generiert und anschließend der cyclische Cluster $\left[\mathrm{Mo}_{12} \mathrm{~S}_{12} \mathrm{O}_{12}(\mathrm{OH})_{12}\left(\mathrm{H}_{2} \mathrm{O}\right)_{6}\right]$ gebildet [1a]. In zwei Fällen konnte ein Polyoxomolybdat(V) Komplex mit einem im Hohlraum gebundenen Oxalat strukturell belegt werden, wobei in beiden Fällen Ammoniumionen als Gegenionen verwendet wurden. So erfolgte die Synthese des Molybdän(V)-oxalat Clusters $\left[\left(\mathrm{n}-\mathrm{C}_{4} \mathrm{H}_{9}\right)_{4} \mathrm{~N}\right]_{2}\left[\mathrm{Mo}_{8} \mathrm{O}_{16}\left(\mathrm{OCH}_{3}\right)_{8}\left(\mathrm{C}_{2} \mathrm{O}_{4}\right)\right]$ durch Umsetzung des Polyoxomolybdat(VI)-Komplexes $\alpha-\left[\left(n-\mathrm{C}_{4} \mathrm{H}_{9}\right)_{4} \mathrm{~N}\right]_{4}\left[\mathrm{Mo}_{8} \mathrm{O}_{26}\right]$ mit Rhodizonsäure $\mathrm{C}_{6} \mathrm{H}_{2} \mathrm{O}_{6} \times 2 \mathrm{H}_{2} \mathrm{O}$. Als Reaktionsmechanismus wird angenommen, dass die Carbonyl-Insertion mit einer Spaltung des Liganden und mit Redoxprozessen gekoppelt ist [4]. Der analoge oktanukleare Polyoxoalkoxyvanadat(IV)Cluster wurde ebenfalls aus Rhodizonsäure mit der Vanadiumverbindung [( $n$ $\left.\mathrm{C}_{4} \mathrm{H}_{9}\right)_{4} \mathrm{~N}_{3}\left[\mathrm{H}_{3} \mathrm{~V}_{10} \mathrm{O}_{28}\right]$ erhalten [5]. Der Polyoxothiomolybdat-Komplex $\left[\mathrm{H}_{2} \mathrm{~N}\left(\mathrm{CH}_{3}\right)_{2}\left(\mathrm{H}_{2} \mathrm{O}\right)\right]\left[\mathrm{Mo}_{8} \mathrm{~S}_{4} \mathrm{O}_{12}(\mathrm{OH})_{8}\left(\mathrm{C}_{2} \mathrm{O}_{4}\right)\right] \times 5.5 \mathrm{H}_{2} \mathrm{O}$, der ebenfalls ein im Hohlraum gebundenes Oxalation aufweist, ist ausgehend von einer Mischung aus $\mathrm{Na}_{2} \mathrm{MoO}_{4} \times \mathrm{H}_{2} \mathrm{O}$, $\mathrm{La}\left(\mathrm{NO}_{3}\right)_{3}, \mathrm{NH}_{2} \mathrm{NH}_{2} \times \mathrm{H}_{2} \mathrm{SO}_{4}, \mathrm{Na}_{2}\left(\mathrm{C}_{2} \mathrm{O}_{4}\right)$, DMSO und $\mathrm{H}_{2} \mathrm{O}$ in einer Hydrothermal-Reaktion zugänglich. Der geschlossene Ring besteht aus insgesamt vier $\left[\mathrm{Mo}_{2} \mathrm{SO}_{3}\right]$-Bausteinen [6].

Wir stellen eine Reaktion vor, bei der ausgehend von oxalatverbrücktem tetranuklearem Molybdän(II)-carboxylat $\left[\mathrm{Mo}_{2}\left(\mathrm{O}_{2} \mathrm{C}-t-\mathrm{Bu}\right)_{3}\right]_{2}\left(\mu-\mathrm{C}_{2} \mathrm{O}_{4}\right)$ und $\mathrm{Mnl}_{2}(\mathrm{THF})_{2}$ in Methanol in Gegenwart von Luft der Polyoxomolybdat(V)-cluster $\left[\mathrm{Mn}\left(\mathrm{CH}_{3} \mathrm{OH}\right)_{6}\right]\left[\mathrm{Mo}_{8} \mathrm{O}_{16}\left(\mathrm{OCH}_{3}\right)_{8}\left(\mathrm{C}_{2} \mathrm{O}_{4}\right)\right]$ erhalten wurde, der somit den ersten Vertreter dieser Polyoxomolybdate mit einem komplexen Gegenion darstellt. 


\section{Experimenteller Teil}

\section{Materialien und Meßmethoden}

Die Synthese der Ausgangsstoffe $\left[\mathrm{Mo}_{2}\left(\mathrm{O}_{2} \mathrm{C}-t-\mathrm{Bu}\right)_{3}\right]_{4}$ und $\mathbf{1}$ wurde unter Argon nach Literaturvorschriften durchgeführt [7, 13]. Die verwendeten Lösungsmittel wurden vor Gebrauch getrocknet und destilliert [8]. Die IR Spektren wurden als $\mathrm{KBr}$ - Preßlinge im Bereich von $400 \mathrm{~cm}^{-1}-4000 \mathrm{~cm}^{-1}$ mit einem Perkin Elmer Gerät System 2000 registriert. UV/Vis-Spektren wurden mit einem Specord S 600 der Fa. Analytik Jena unter Verwendung einer methanolischen Lösung in Quarzküvetten erhalten. ESR-Spektren wurden mit einem Bruker ESP 300E X-Band Spektrometer aufgenommen. Die Simulation der ESR Spektren erfolgte mit Hilfe des Programms XSophe Computer Simulation Software Suite, Version 1.1.4 Copyright 1993--2004, entwickelt im Centre for Magnetic Resonance and Department of Mathematics, The University of Queensland, Brisbane, Queensland, Australia 4072 und Bruker Biospin (früher Bruker Analytik), Rheinstetten, Germany, GmbH. Die Thermoanalyse von 2 wurde an einem Netzsch STA 429 in einem Pt-Ir-Tiegel bei 298-1573 K mit einer Aufheizrate von $5 \mathrm{~K} / \mathrm{min}$ durchgeführt. Massenspektren wurden unter Verwendung eines Finnigan MAT SSQ 710 Gerätes erhalten. Die Elementaranalyse wurde in einem Labor des Institutes für Organische Chemie und Makromolekulare Chemie der Friedrich-SchillerUniversität Jena durchgeführt. Messungen der magnetischen Suszeptibilität wurden mit einer gepulverten Probe mittels eines Quantum-Design MPMSR-5S SQUID-Magnetometers, das mit einem 5 Tesla Magneten ausgerüstet ist, in einem Temperaturbereich von 2 bis $300 \mathrm{~K}$ und einem angelegten magnetischen Feld von 2000 Oe durchgeführt. Die Probe wurde in einer Gelatine-Kapsel präpariert und in einem nicht-magnetischen Probenhalter fixiert. Die gemessenen primären Magnetisierungsdaten wurden für hierdurch verursachte Effekte korrigiert. Die Korrektur der hieraus erhaltenen Suszeptibilitätsdaten für den Diamagnetismus der Probe selbst wurde unter Anwendung von Pascal-Konstanten nach Inkrementmethoden durchgeführt [9].

Kristallstrukturuntersuchung. Die Strukturuntersuchung von 2 wurde auf einem Enraf Nonius Kappa CCD Diffraktometer, Abstand Kristall-Detektor $29 \mathrm{~mm}$, mittels monochromatischer Mo- $K_{\alpha}$ Strahlung durchgeführt. Der Kristall wurde in einem Strom kalten Stickstoffs montiert. Die Meßdaten wurden im Hinblick auf Lorentz- und Polarisationseffekte korrigiert, eine Absorptionskorrektur wurde nicht durchgeführt [10]. Die Struktur wurde mittels direkter Methoden gelöst und nach der Methode der kleinsten Fehlerquadrate gegen $\mathrm{F}^{2}$ verfeinert (SHELXS86 und SHELXL97) [11]. Die Abbildungen der Molekülstrukturen wurden mit dem Programm XP erstellt [12]. Zusätzliche Informationen zu den Kristallstrukturuntersuchungen sind beim Cambridge Crystallographic Data Centre unter Angabe der Hinterlegungsnummer CCDC-690768 erhältlich. 
Kristalldaten für $\left[\mathrm{Mn}\left(\mathrm{CH}_{3} \mathrm{OH}\right)_{6}\right]\left[\mathrm{Mo}_{8} \mathrm{O}_{16}\left(\mathrm{OCH}_{3}\right)_{8}\left(\mathrm{C}_{2} \mathrm{O}_{4}\right)\right] \times 8 \mathrm{CH}_{3} \mathrm{OH}$, 2: monoklin, Raumgruppe $\mathrm{P} 22_{1} / \mathrm{c}, a=13.529(3), b=15.481(3), c=14.622(3) \AA, \beta=93.97(3)^{\circ}, V=3055(1) \AA^{3}, T=183$ $\mathrm{K}, Z=2, \rho_{\text {ber }}=2.026 \mathrm{~g} \mathrm{~cm}^{-3}, \mu\left(\mathrm{Mo}_{\mathrm{K} \alpha}\right)=0.71073 \mathrm{~nm}, 20182$ gemessene Reflexe $\left(\mathrm{R}_{\text {int }}=\right.$ 0.0450), 6952 unabhängige Reflexe, 5717 beobachtete Reflexe $(I>2 \sigma(I))$, Kristallabmessungen $0.05 \times 0.05 \times 0.04 \mathrm{~mm}^{3}, 364$ Parameter, $R_{1}=0.0475, w_{2}=0.1186$, $\mathrm{GooF}=1.130$.

$\left[\mathrm{Mo}_{2}\left(\mathrm{O}_{2} \mathrm{C}^{\mathrm{t}} \mathrm{Bu}\right)_{3}\right]_{2}\left(\boldsymbol{C}_{2} \mathrm{O}_{4}\right)$ (1) [13]: In einem $100 \mathrm{ml}$ Rundkolben werden 1,0 g (1,68 mmol) $\left[\mathrm{Mo}_{2}\left(\mathrm{O}_{2} \mathrm{C}-{ }^{\mathrm{t}} \mathrm{Bu}\right)_{4}\right]$ und $0,0755 \mathrm{~g}(0,84 \mathrm{mmol})$ Oxalsäure in $20 \mathrm{ml}$ Toluol gegeben. Diese gelbe Reaktionsmischung wird bei Raumtemperatur 3 Tage gerührt. Während des Rührens ist die Bildung eines roten Feststoffes zu beobachten. Der rote Niederschlag wird abfiltriert und anschließend $3 x$ mit $20 \mathrm{ml}$ Toluol und $3 x$ mit $25 \mathrm{ml}$ Hexan gewaschen und im Vakuum getrocknet. Ausbeute: 0,859g (95\%).

$\left[\mathrm{Mn}\left(\mathrm{CH}_{3} \mathrm{OH}\right)_{6}\right]\left[\mathrm{Mo}_{8} \mathrm{O}_{16}\left(\mathrm{OCH}_{3}\right)_{8}\left(\mathrm{C}_{2} \mathrm{O}_{4}\right)\right] \times 8 \mathrm{CH}_{3} \mathrm{OH}(2)$ : In einem $50 \mathrm{ml}$ Rundkolben werden $0,031 \mathrm{~g}(0,028 \mathrm{mmol}) 1 \mathrm{in} 5 \mathrm{ml}$ THF gelöst und $0,050 \mathrm{~g}(0,111 \mathrm{mmol})\left[\mathrm{Mnl}_{2}(\mathrm{thf})_{2}\right]$ in $5 \mathrm{ml}$ THF zugegeben. Die rotbraune Lösung wird 24 Stunden bei Raumtemperatur gerührt. Das Lösungsmittel wird im Vakuum abgezogen, mit $5 \mathrm{ml} \mathrm{MeOH}$ versetzt und nach anschließender Sauerstoffzufuhr bei $-20{ }^{\circ} \mathrm{C}$ aufbewahrt. Nach ca. 2 Wochen kann ein roter kristalliner Feststoff isoliert werden. Ausbeute: 0,0081g (18\%). Mikro-ESI in Methanol: $\mathrm{m} / \mathrm{z}=$ $1359\left(\mathrm{Mo}_{8} \mathrm{O}_{16}(\mathrm{OMe})_{8}\left(\mathrm{C}_{2} \mathrm{O}_{4}\right), 2\right), 680\left(\mathrm{Mo}_{8} \mathrm{O}_{16}(\mathrm{OMe})_{8}\left(\mathrm{C}_{2} \mathrm{O}_{4}\right) / 2,100\right), 255\left(\mathrm{Mo}_{2} \mathrm{O}_{4}{ }^{+}, 3\right)$; IR $(\mathrm{KBr})$ : $v(\mathrm{CH}) 2965 \mathrm{w}, 2931 \mathrm{w}, 2829 \mathrm{w}, \mathrm{v}(\mathrm{CO}) 1664 \mathrm{vs}) \mathrm{v}(\mathrm{Mo}=\mathrm{O}) 969 \mathrm{vs}, \mathrm{v}$ (Mo-O-Mo) $815 \mathrm{~m} \mathrm{~cm}^{-1}$; UV/VIS: $\lambda_{\max }(\lg \varepsilon): 223 \mathrm{~nm}(4,117), 238 \mathrm{~nm}(4,188), 260 \mathrm{~nm}(3,548), 490 \mathrm{~nm}(4,752)$; ESR (Methanol, $298 \mathrm{~K}$ ): I = 5/2, $\mathrm{g}=2,007 ; \mathrm{A}=9 \mathrm{mT}$; Elementaranalyse für $\mathrm{C}_{24} \mathrm{H}_{80} \mathrm{O}_{42} \mathrm{MnMo}_{8}$ $\left(1863,33 \mathrm{gmol}^{-1}\right)$ : C 14,88 (ber. 15,46); H 4,21 (4,29).

\section{Ergebnisse und Diskussion}

Der dinukleare Molybdäncarboxylat-Komplex $\left[\mathrm{Mo}_{2}\left({ }^{t} \mathrm{BuCOO}\right)_{4}\right]$ mit einer formalen MolybdänMolybdän-Vierfachbindung wird mit Oxalsäure versetzt, wodurch nach dreitägigem Rühren der oxalatverbrückte Molybdänkomplex 1 erhalten wird [13]. Ausgehend von 1 konnte der luftstabile Polyoxomolybdat(V)-Cluster 2 durch Zugabe von $\left[\mathrm{Mnl}_{2}(\mathrm{THF})_{2}\right]$ im Verhältnis von 1:4 synthetisiert werden (Schema 1), wobei 2 in Form roter Kristalle anfällt. Die Reaktion gelingt bis zu einem Verhältnis 1 : $\mathrm{Mnl}_{2}$ von 1:2, verläuft jedoch unter Verwendung eines vierfachen Überschusses in Bezug auf die Reaktionszeit und Ausbeute am besten. Unter streng inerten Reaktionsbedingungen kann keine analoge Reaktion bobachtet werden. Weitere orientierende Versuche haben gezeigt, dass die Reaktion bei Verwendung von 

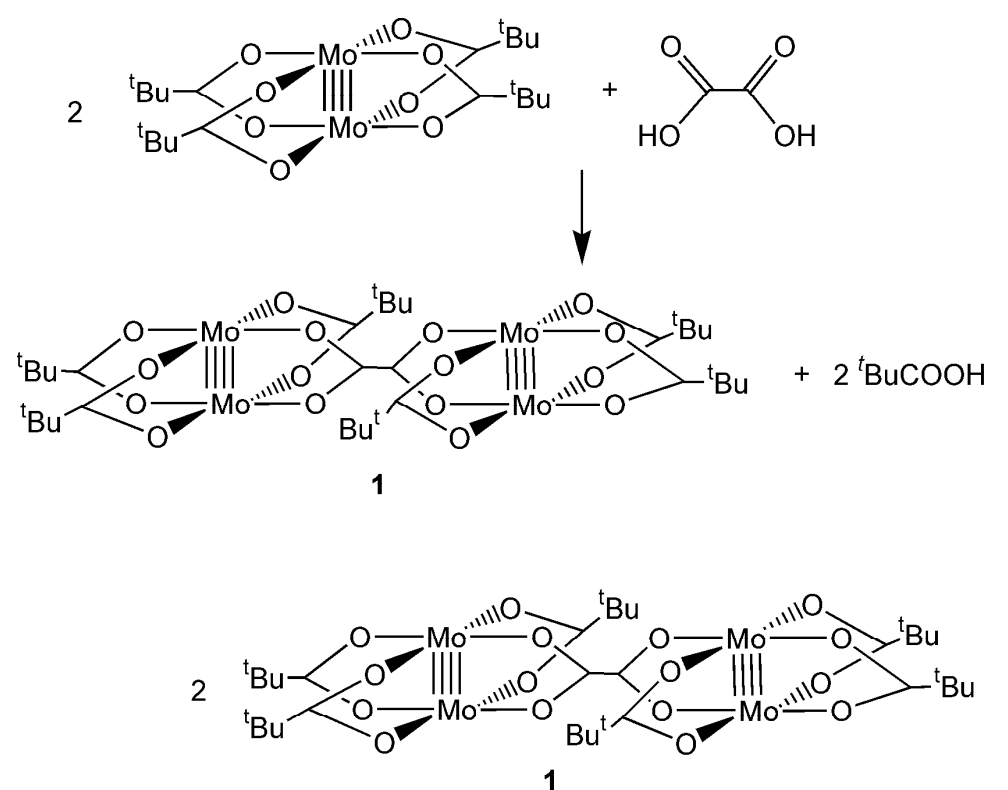

$$
\begin{array}{l|l}
+4 \mathrm{Mnl}_{2}(\mathrm{THF})_{2} & -8{ }^{t} \mathrm{BuCOOH} \\
+8 \mathrm{CH}_{3} \mathrm{OH} & -3 \mathrm{I}_{2} \\
+11 \mathrm{O}_{2} & -\mathrm{Mn}\left(\mathrm{IO}_{3}\right)_{2} \\
& -2 \mathrm{Mn}\left({ }^{\mathrm{t}} \mathrm{BuCOO}\right) \\
& -2 \mathrm{CO}_{2}
\end{array}
$$
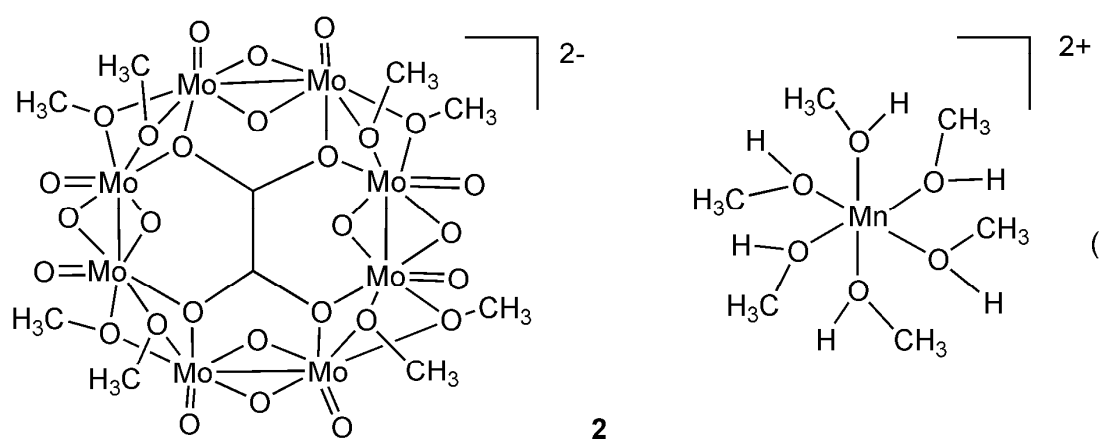

$\mathrm{MnCl}_{2}$ nicht stattfindet, mit anderen Metalliodiden wie z.B. Nal, KI oder $\mathrm{Sbl}_{3}$ jedoch ebenfalls zur Bildung des Polyoxomolybdats führt.

Der Reaktionsablauf stellt offensichtlich eine komplexe Redoxreaktion dar, in der sowohl die Molybdänzentren in $\mathbf{1}$ als auch die lodidionen und vermutlich eines der Oxalationen oxidiert werden. Die Bildung von lodat sowie von elementarem lod kann durch klassische qualitative Nachweismethoden gezeigt werden [14]. Die Oxidationsäquivalente stammen dabei offensichtlich von den in den lodationen wie auch im Anion von 2 inkorporierten Sauerstoffatomen und damit aus Luftsauerstoff. Eine mögliche Deutung des Reaktionsablaufs in Bezug auf die Redoxprozesse ist in Schema 2 dargestellt.

Die Bildung von Polyoxomolybdaten vom Typ 2 kann durch typische IR-Banden gezeigt werden. So weist das IR-Spektrum der Verbindung 2 eine Bande bei $815 \mathrm{~cm}^{-1}$ auf, welche den verbrückenden Oxogruppen (Mo-O-Mo) zugeordnet werden kann, während eine Bande bei $969 \mathrm{~cm}^{-1}$ die $v(\mathrm{Mo}=0)$-Streckschwingung anzeigt. Ausserdem zeigt das Spektrum eine Bande bei $2931 \mathrm{~cm}^{-1}$ für $v(\mathrm{CH})$ sowie bei $1664 \mathrm{~cm}^{-1}$ für $v(\mathrm{C}=\mathrm{O})$. 


$$
\begin{aligned}
& 8 \mathrm{Mo}^{2+} \longrightarrow 2 \mathrm{Mo}^{5+}+24 \mathrm{e}^{-} \\
& 8 \mathrm{I}^{-}+3 \mathrm{O}_{2} \longrightarrow 2 \mathrm{IO}_{3}^{-}+3 \mathrm{I}_{2}+6 \mathrm{e}^{-} \\
& \mathrm{C}_{2} \mathrm{O}_{4}^{2-} \longrightarrow 2 \mathrm{CO}_{2}+2 \mathrm{e}^{-} \\
& 8 \mathrm{O}_{2}+32 \mathrm{e}^{-} \longrightarrow 16 \mathrm{O}^{2-}
\end{aligned}
$$

\section{Molekülstruktur von $\left[\mathrm{Mn}\left(\mathrm{CH}_{3} \mathrm{OH}\right)_{6}\right]\left[\mathrm{Mo}_{8} \mathrm{O}_{16}\left(\mathrm{OCH}_{3}\right)_{8}\left(\mathrm{C}_{2} \mathrm{O}_{4}\right)\right] \times 8 \mathrm{CH}_{3} \mathrm{OH}(2)$}

Die Molekülstruktur des Polyoxomolybdatanions wird in Abbildung 1 gezeigt, ausgewählte Bindungslängen und -winkel sind in Tabelle 1 zusammengefasst. Der Aufbau dieser Einheit entspricht bereits früher strukturanalytisch untersuchten Derivaten [4, 6]. Das Anion enthält insgesamt 8 Molybdänatome in jeweils stark verzerrt oktaedrischer Koordinationsumgebung. Die Molybdänzentren weisen eine cyclische Anordnung auf, in der die Metallatome abwechselnd über zwei Oxo- bzw. zwei Methoxoeinheiten verbrückt werden. Dabei sind die Mo-Mo-Abstände der über die $\mu$-Oxo-Einheiten verbrückten Molybdänzentren ca. 70 pm kürzer als die durch Methoxobrücken realisierten Abstände. Jedes Molybdänatom weist außerdem einen terminalen Oxoliganden auf, wobei die kurzen Molydän-Sauerstoffabstände den formalen Doppelbindungscharakter belegen. In der Mitte des Ringes, die gleichzeitig auch ein kristallografisches Inversionszentrum darstellt, befindet sich ein Oxalation, dessen Kohlenstoffatom C1 statistisch fehlgeordnet ist.

Im Gegensatz zu den bisher bekannten Derivaten mit quartären Ammoniumionen ist in der Kristallstruktur von 2 auch das Gegenion zum Polyoxomoybdatanion ein komplexes Ion, dessen Struktur in Abbildung 2 gezeigt ist. Es handelt sich um ein nahezu ideal oktaedrisch

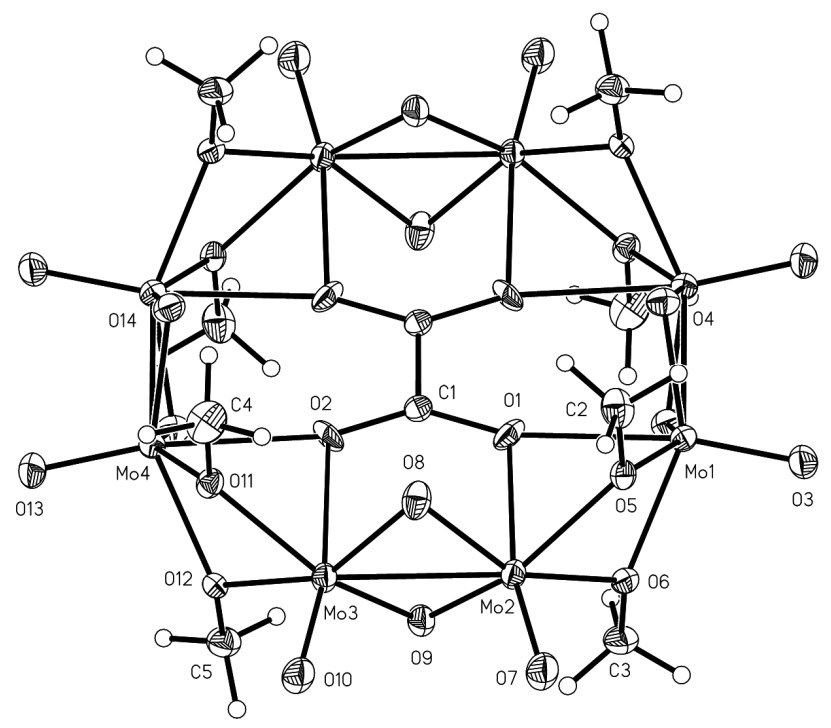

Abb. 1. Molekülstruktur des Polyoxomolybdat-Anions in 2. Die thermischen Ellipsoide repräsentieren eine Wahrscheinlichket von 40\%, die Fehlordnung von C1 ist der Übersichtlichkeit halber nicht eingezeichnet. 


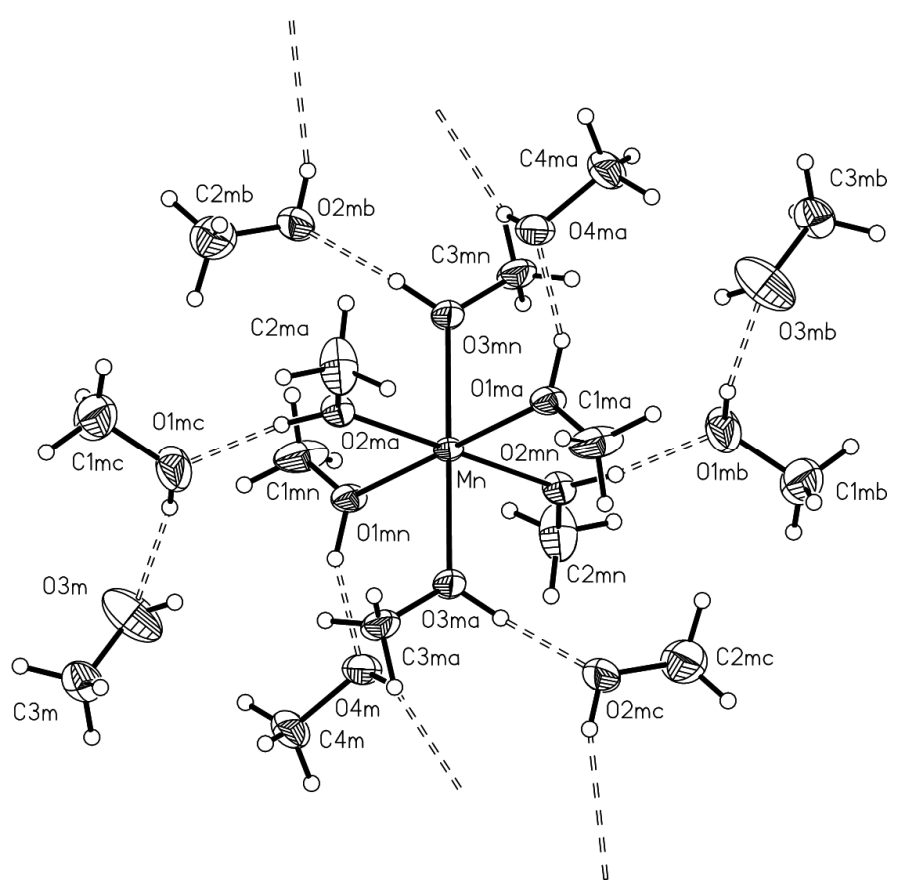

Abb. 2. Molekülstruktur des $\left[\mathrm{Mn}\left(\mathrm{CH}_{3} \mathrm{OH}\right)_{6}\right]^{2+}$ Kations in 2. Die thermischen Ellipsoide repräsentieren eine Wahrscheinlichkeit von $40 \%$. Eingezeichnet sind außerdem die über Wasserstoffbrückenbindungen fixierten Solvensmoleküle, freie gestrichelte Bindungen stellen Wasserstoffbrücken zu terminalen Oxoliganden benachbarter Polyoxomolybdat-Anionen dar.

Tabelle 1. Ausgewählte Bindungslängen [pm] und Bindungswinkel [ ${ }^{\circ}$ von 2.

\begin{tabular}{|c|c|c|c|c|c|c|c|}
\hline Mo1-Mo2 & $3.250(1)$ & Mo2-Mo3 & $2.5810(9)$ & Mo3-Mo4 & $3.257(1)$ & Mo1-Mo4 & $2.5734(8)$ \\
\hline Mo1-O1 & $237.9(4)$ & Mo1-O3 & $168.4(4)$ & Mo3-O11 & $208.7(4)$ & Mo3-O12 & $208.5(4)$ \\
\hline Mo1-O4 & $192.9(4)$ & Mo1-O5 & $209.7(4)$ & Mo4-O2 & $240.9(4)$ & Mo4-O11 & $210.2(4)$ \\
\hline Mo1-O6 & $209.7(4)$ & Mo1-O14 & $192.8(4)$ & Mo4-O12 & $210.0(4)$ & Mo4-O13 & $168.2(4)$ \\
\hline Mo2-O1 & $234.3(4)$ & Mo2-O5 & $208.9(4)$ & Mo4-O14 & $193.7(4)$ & Mo4-O4 & $193.4(4)$ \\
\hline Mo2-O6 & $208.9(4)$ & Mo2-O7 & $168.4(4)$ & C1-C1a & $149(1)$ & C1-O1 & $132(1)$ \\
\hline Mo2-O8 & 195.1(4) & Mo2-O9 & $192.2(4)$ & $\mathrm{C} 1-\mathrm{O} 2$ & $131(1)$ & Mn-O1mn & $216.9(5)$ \\
\hline Mo3-O2 & $235.8(4)$ & Mo3-O8 & $194.6(4)$ & $\mathrm{Mn}-\mathrm{O} 2 \mathrm{mn}$ & $215.1(5)$ & $\mathrm{Mn}-\mathrm{O} 3 \mathrm{mn}$ & $218.1(5)$ \\
\hline Mo3-O9 & 193.5(4) & Mo3-O10 & $167.1(4)$ & & & & \\
\hline O1-Mo1-O3 & $167.7(2)$ & O1-Mo1-O4 & $80.8(2)$ & O8-Мо3-O10 & $105.3(2)$ & O8-Мo3-O11 & $91.0(2)$ \\
\hline O1-Mo1-O5 & $68.2(2)$ & O1-Mo1-O6 & $68.9(2)$ & O8-Мо3-O12 & $150.1(2)$ & O9-Мо3-O10 & $106.3(2)$ \\
\hline O1-Mo1-O14 & $82.2(2)$ & O3-Mo1-O4 & $107.1(2)$ & O9-Мо3-O11 & $149.6(2)$ & O9-Мo3-O12 & $90.5(2)$ \\
\hline O3-Mo1-O5 & $101.9(2)$ & O3-Mo1-O6 & $101.5(2)$ & O10-Mo3-O11 & 101.0(2) & O10-Mo3-O12 & 101.6(2) \\
\hline O3-Mo1-O14 & $106.0(2)$ & O4-Mo1-O5 & $89.6(2)$ & O11-Mo3-O12 & $70.9(2)$ & O2-Mo4-O11 & $68.8(2)$ \\
\hline O4-Mo1-O6 & $148.3(2)$ & O4-Mo1-O14 & $94.7(2)$ & O2-Mo4-O12 & $68.8(1)$ & O2-Mo4-O13 & $168.5(2)$ \\
\hline O5-Mo1-O6 & $71.1(2)$ & O5-Mo1-O14 & $149.1(2)$ & O2-Mo4-O14 & $80.1(2)$ & O2-Mo4-O4 & $81.1(2)$ \\
\hline O6-Mo1-O14 & $90.3(2)$ & O1-Mo2-O5 & $69.1(2)$ & O11-Mo4-O12 & $70.35(2)$ & O11-Mo4-O13 & $101.7(2)$ \\
\hline O1-Mo2-O6 & $69.7(2)$ & O1-Mo2-O7 & $168.0(2)$ & O11-Mo4-O14 & $90.7(2)$ & O11-Mo4-O4 & $148.1(2)$ \\
\hline O1-Mo2-O8 & $82.6(2)$ & O1-Mo2-O9 & $81.4(2)$ & O12-Mo4-O13 & $102.4(2)$ & O12-Mo4-O14 & $147.7(2)$ \\
\hline O5-Mo2-O6 & $71.4(2)$ & O5-Mo2-O7 & $101.2(2)$ & O12-Mo4-O4 & $89.7(2)$ & O13-Mo4-O14 & $107.1(2)$ \\
\hline O5-Mo2-O8 & $90.9(2)$ & O5-Mo2-O9 & $148.8(2)$ & O13-Mo4-O4 & $106.8(2)$ & O14-Mo4-O4 & $94.2(2)$ \\
\hline O6-Mo2-O7 & $101.0(2)$ & O6-Mo2-O8 & $150.9(2)$ & Mo1-O1-Mo2 & $87.0(1)$ & Mo1-O1-C1 & $161.4(6)$ \\
\hline O6-Mo2-O9 & $89.9(2)$ & O7-Mo2-O8 & $105.1(2)$ & Mo2-O1-C1 & $111.6(6)$ & Mo3-O2-Mo4 & $86.2(2)$ \\
\hline O7-Mo2-O9 & $106.7(2)$ & O8-Mo2-O9 & $94.8(2)$ & Mo3-O2-C1 & $112.1(6)$ & Mo4-O2-C1 & $161.7(6)$ \\
\hline O2-Мо3-O8 & $81.6(2)$ & O2-Мo3-O9 & $81.2(2)$ & $\mathrm{O} 1-\mathrm{C} 1-\mathrm{O} 2$ & $139(1)$ & O1-C1-C1a & $110(1)$ \\
\hline O2-Mo3-O10 & $169.1(2)$ & O2-Mo3-O11 & $70.0(2)$ & $\mathrm{O} 2-\mathrm{C} 1-\mathrm{C} 1 \mathrm{a}$ & $111(1)$ & $\mathrm{O} 1 \mathrm{mn}-\mathrm{Mn}-\mathrm{O} 2 \mathrm{mn}$ & $93.9(2)$ \\
\hline O2-Mo3-O12 & $70.1(1)$ & O8-Mo3-O9 & $94.5(2)$ & O1mn-Mn-O3mn & $93.8(2)$ & $\mathrm{O} 2 \mathrm{mn}-\mathrm{Mn}-\mathrm{O} 3 \mathrm{mn}$ & $92.2(2)$ \\
\hline
\end{tabular}


von sechs Methanolmolekülen koordiniertes Manganion, wobei das Manganatom selbst auf einem kristallografischen Inversionszentrum liegt. Die Methanolliganden des Kations wirken als Wasserstoffbrückenbindungs-Donoren bezüglich Solvens-Methanolmolekülen in der Elementarzelle. Die OH-Gruppen dieser Solvensmoleküle agieren wiederum als H-BrückenDonoren gegenüber terminalen Oxoliganden der Polyoxomolybdatanionen $(\mathrm{O} 2 \mathrm{~m}, \mathrm{O} 4 \mathrm{~m})$ oder gegenüber einem weiteren Solvensmolekül (O1m).

Abbildung 3 zeigt die Auftragung der molaren Suszeptibilität gegen die inverse Temperatur für das Polyoxomolybdat 2. Aus der berechneten Ausgleichsgeraden einhält man eine CurieKonstante von $C=4,88(1) \mathrm{cm}^{-3} \mathrm{~K} \mathrm{~mol}^{-1}$, die in guter Näherung dem Paramagnetismus eines Systems mit $S=5 / 2$ entspricht, wie man es für ein isoliertes high-spin Mn(II)-lon erwarten würde [15]. Dies ist im Einklang mit der beobachteten Bildung von Metall-Metall-Bindungen in zweikernigen $\mathrm{Mo}(\mathrm{V})$-Einheiten in vollständig reduzierten Polyoxometalaten(V) [2a]. Konsequenterweise tragen die Mo(V)-Einheiten auch nicht zum Paramagnetismus der Verbindung 2 bei. Interessanterweise zeigt Verbindung 2 nach Korrektur des zu erwartenden Diamagnetismus (siehe Experimenteller Teil) einen deutlichen temperaturunabhängigen paramagnetischen Anteil für die gemessene Suszeptibilität. Dieser temperaturunabhängige Paramagnetismus ist jedoch nicht unerwartet für Polyoxomolybdate [16].

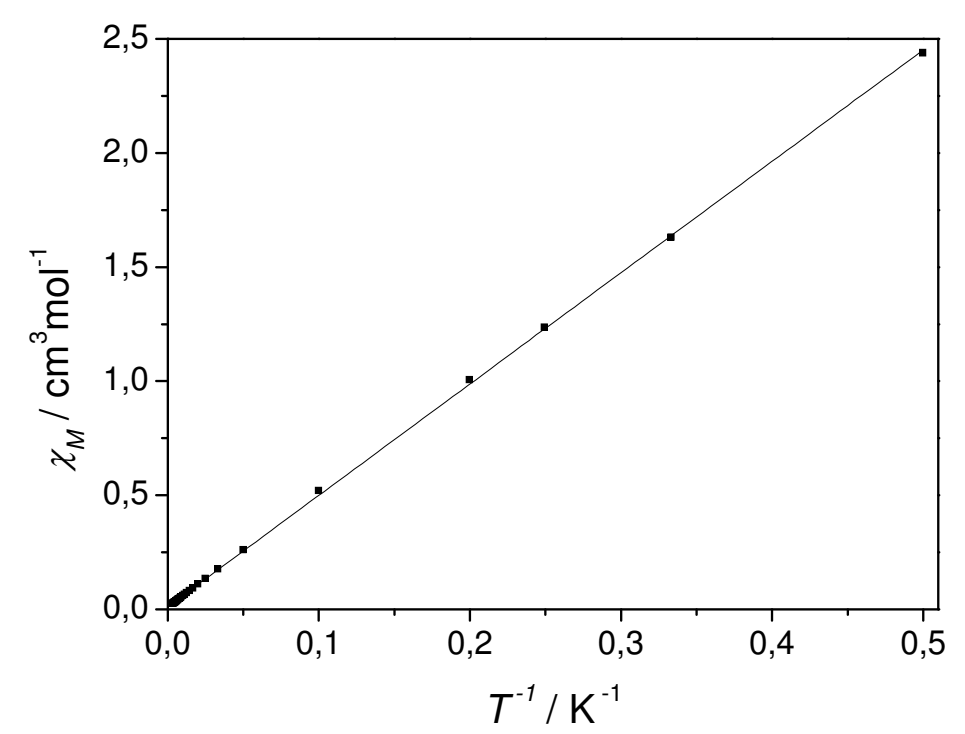

Abb. 3. Molare magnetische Suszeptibilität als Funktion der inversen Temperatur für 2. 
Abbildung 4 zeigt das gemessene ESR-Spektrum von 2 in methanolischer Lösung bei Raumtemperatur. Im Spektrum ist erwartungsgemäß das isotrope Signal einer isolierten $\mathrm{Mn}(\mathrm{II})$-Spezies mit einer Hyperfeinaufspaltung in sechs Linien (Kernspin Mn: $I=5 / 2$ ) zu beobachten, wobei die in der Simulation verwendeten Werte (isotroper $g$-Wert $g_{\text {iso }}=1.999$ und isotrope Hyperfeinstukturkonstante $A_{\text {iso }}=95 \mathrm{G}$ ) den Erwartungen entsprechen.[17]

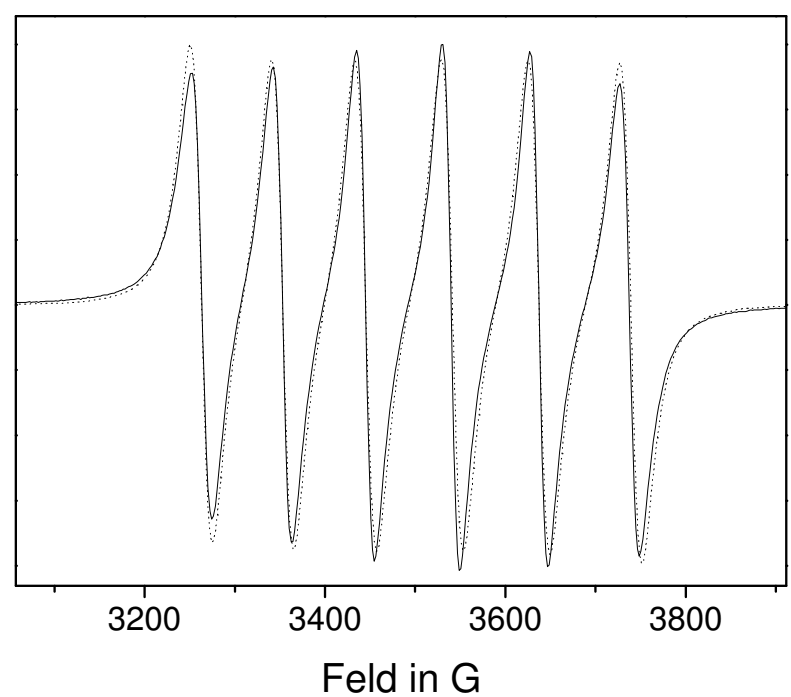

Abb. 4. ESR-Spektrum von 2 in Methanol bei Raumtemperatur; die durchgezogene Linie stellt das gemessene Spektrum dar, die gepunktete das simulierte mit giso $=1.999$ und $A_{\text {iso }}=95 \mathrm{G}$.

Eine Untersuchung von 2 mittels Thermogravimetrie (Abbildung als Zusatzmaterial) zeigt bis ca. $400^{\circ} \mathrm{C}$ die sukzessive Abspaltung der sechs Methanol-Liganden sowie der acht Methanolmoleküle, die im Kristallgitter eingelagert sind, und des Oxalatliganden, der vermutlich in Form von zwei Formeleinheiten $\mathrm{CO}_{2}$ eliminiert wird. Daraus ergibt sich ein Massenverlust von ca. 30\%. Die verbleibenden Spezies erleiden oberhalb von ca. $700^{\circ} \mathrm{C}$ einen weiteren Masseverlust von ca. 59\% relativ zur Masse von 2 . Als Rückstand verbleiben grünliche Kristalle, die vermutlich $\mathrm{MnO}$ darstellen und eine farblose kristalline Verbindung (vermutlich $\mathrm{MoO}_{3}$ ). Da $\mathrm{MoO}_{3}$ unter den Bedingungen der Thermoanalyse zur Sublimation neigt, erklärt sich der hohe Masseverlust bezogen auf die Einwaage von 2 von insgesamt 86\% aus dem teilweisen Verlust des molybdänhaltigen Endproduktes der Thermolyse.

Da Umsetzungen des Molybdän(II)-Komplexes 1 mit anderen Metalliodiden ebenfalls zu Molybdän(V)-Komplexen mit demselben Polyoxomolybdat-Anion wie in $\mathbf{2}$ führen, in diesen Fällen jedoch mit einfach bzw. dreifach positiv geladenen Gegenionen, sind von 2 unterschiedliche Stöchiometrien sowie supramolekulare Architekturen im Festkörper zu erwarten. Die Untersuchung dieser Fragestellungen ist Gegenstand aktueller Arbeiten. 
Die Autoren sind der Deutschen Forschungsgemeinschaft (IM 22/6-2) für die finanzielle Unterstützung der Arbeiten dankbar. Eike T. Spielberg dankt dem Freistaat Thüringen für die Gewährung eines Landesgraduiertenstipendiums sowie der Carl-Zeiss-Stiftung für weitere finanzielle Unterstützung. Ein Dank gilt außerdem Prof. Malcolm Chisholm, Ohio State University, Columbus, $\mathrm{OH}$ für vielfältige Diskussionen.

\section{Literatur}

[1] a) E. Cadot, B. Salignac, S. Halut, F. Sécheresse, Angew. Chem. Int. Ed. Engl. 1998, 37, 611-613; b) B. Modec, J. V. Brečič, R. C. Finn, R. S. Rarig, J. Zubieta, Inorg. Chim. Acta 2001, 322, 113-119; c) S. N. Shaikh, J. Zubieta, Inorg. Chem.. 1986, 25, 46154616, d) A. Müller, E. Krickemeyer, J. Meyer, H. Bögge, F. Peters, W. Plass, E. Diemann, S. Dillinger, F. Nonnenbruch, M. Randerath, C. Menke, Angew. Chem. Int. Ed. Engl. 1995, 34, 2122-2124, e) Md. A. Alam, Y.-S. Kim, S. Ogawa, A. Tsuda, N. Ishii, T. Aida, Angew. Chem. Int. Ed. Engl. 2008, 46, 8198-8202, f) M. T. Pope, A. Müller (Eds.), Polyoxometalates: From Platonic Solids to Anti-retroviral Activity, Kluwer Academic Publichers, Dordrecht, Netherlands, 1994.

[2] a) H. K. Chae, W. G. Klemperer, T. A. Marquart, Coord. Chem. Rev. 1993, 128, 209224; b) A. Dolbecq, L. Lisnard, P. Mialane, J. Marrot, M. Benard, M.M. Rohmer, F. Secheresse, Inorg. Chem. 2006, 45, 5898-5910; c) M. J. Manos, J. D. Woollins, A. M. Z. Slawin, T. A. Kabanos, Angew. Chem. Int. Ed. 2002, 41, 2801-2805; d) A. Müller, S. Dillinger, E. Krickemeyer, H. Bögge, W. Plass, A. Stammler, R. C. Haushalter, Z. Naturforsch. 1997, 52b, 1301-1306; e) A. Müller, E. Krickemeyer, S. Dillinger, H. Bögge, A. Proust, W. Plass, R. Rohlfing, Naturwissenschaften 1993, 80, 560-564.

[3] B. Modec, D. Dolenc, J.V. Brečič, J. Koller, J. Zubieta, Eur. J. Inorg. Chem. 2005, 3224-3237.

[4] Q. Chen, S. Liu, J. Zubieta, Angew. Chem. Int. Ed. Engl. 1988, 27, 1724-1725.

[5] Q. Chen, S. Liu, J. Zubieta, Inorg. Chem. 1989, 28, 4433-4434.

[6] X-P Zhan, C-Z. Lu, W-B. Yang, H-W. Ma, C-D. Wu, Q-Z. Zhang, Dalton Trans., 2003, 1457-1458.

[7] A. G. Brignole, F. A. Cotton, Inorg. Synth, 1972, 13, 81.

[8] D. D. Perrin, W. L. F. Armarego, D. R. Perrin, Purification of Laboratory Chemicals, Pergamon Press, New York, 1980.

[9] a) W. Haberditzl, „Magnetochemie“, Akademie-Verlag, 1968; b) W. Haberditzl, Angew. Chem. Int. Ed. Engl. 1966, 5, 288-298.

[10] a) COLLECT, Nonius BV, The Netherlands 1998; b) Z. Otwinowski, W. Minor, Methods in Enymology, Vol. 276, Macromolecular Crystallography Part A, edited by C.W. Carter, R.M. Sweet, Academic Press, New York 1997, 307. 
[11] a) G. Sheldrick, Universität Göttingen, 1986; b) G. Sheldrick, Universität Göttingen, 1997.

[12] Siemens Analytical Xray Inst. Inc., XP - Interactive Molecular Graphics, Vers. 4.2, 1990.

[13] R. H. Cayton, M. H. Chisholm, J. C. Huffmann, E. B. Lobkovsky, J. Am. Chem. Soc. 1991, 113, 8709-8724.

[14] G. Jander, E. Blasius, Lehrbuch der analytischen und präparativen anorganischen Chemie, S. Hirzel Verlag Stuttgart, 16. Auflage, 2006.

[15] H. Lueken, Magnetochemie, Teubner Studienbücher 1999.

[16] D. Gatteschi, R. Sessoli, W. Plass, A. Müller, E. Krickemeyer, J. Meyer, D. Sölter, P. Adler, Inorg. Chem. 1996, 35, 1926-1934.

[17] A. Abragam, B. Bleaney, Electron Paramagnetic Resonance of Transition Ions, Oxford University Press, England 1975. 


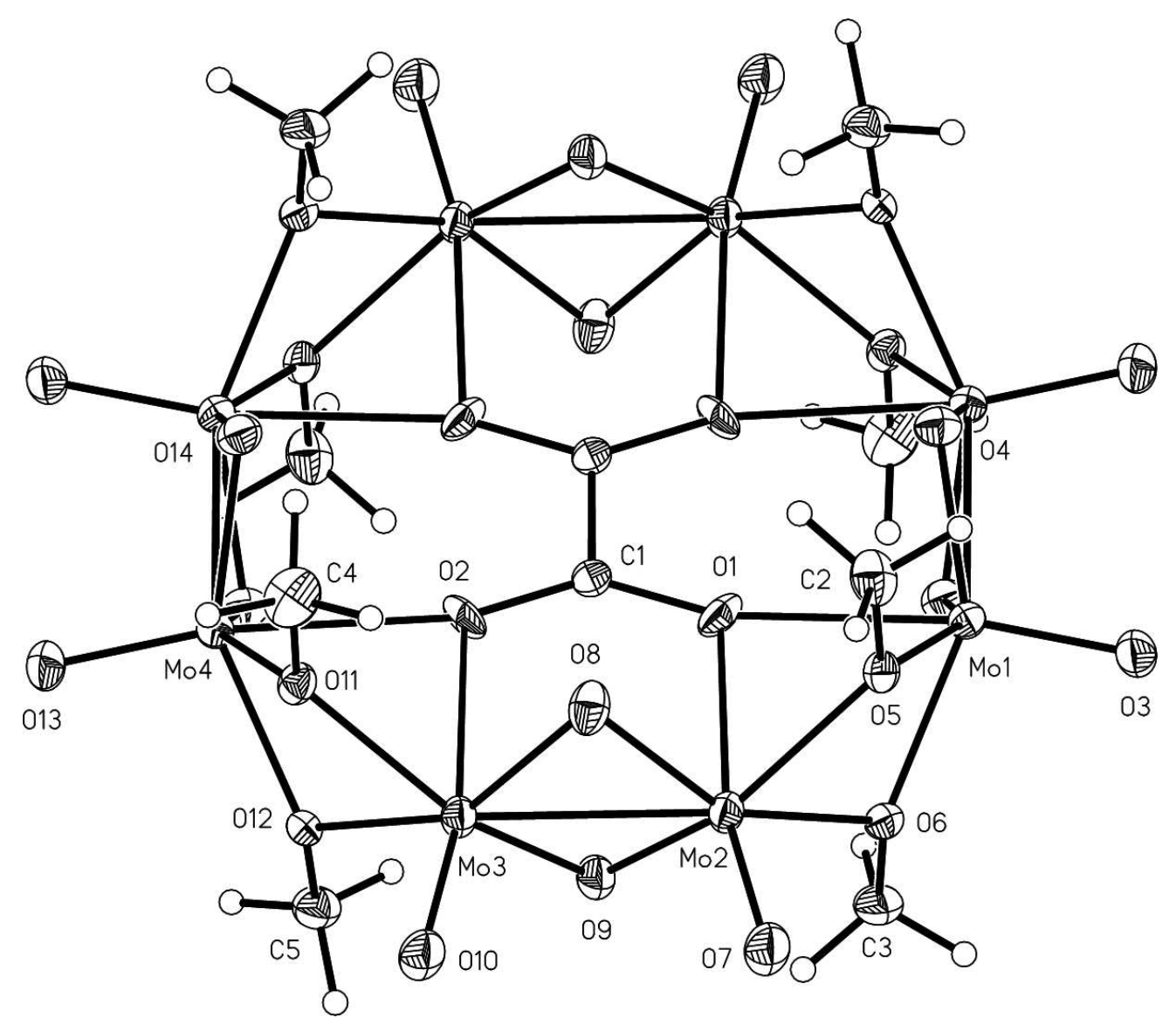




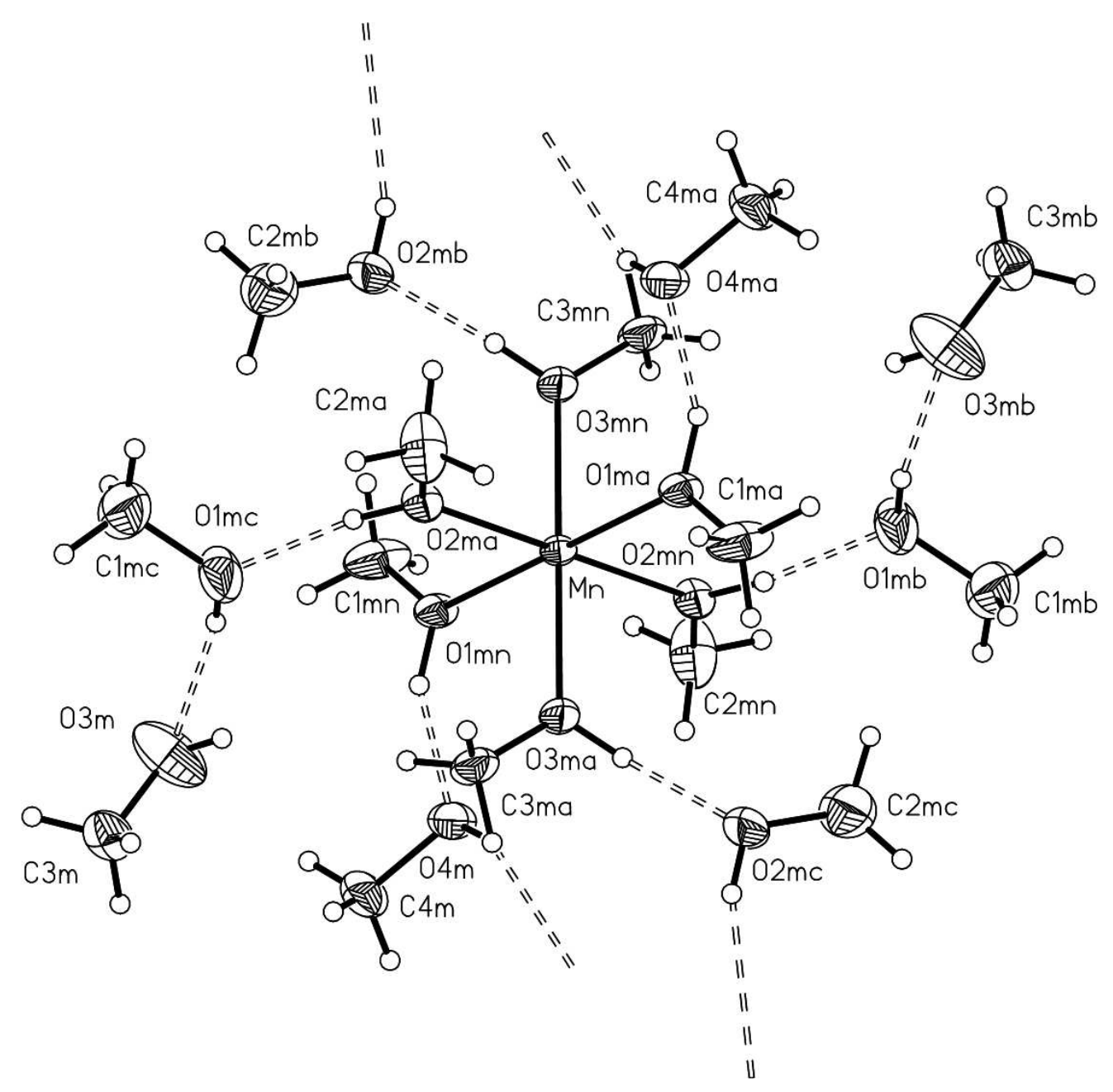




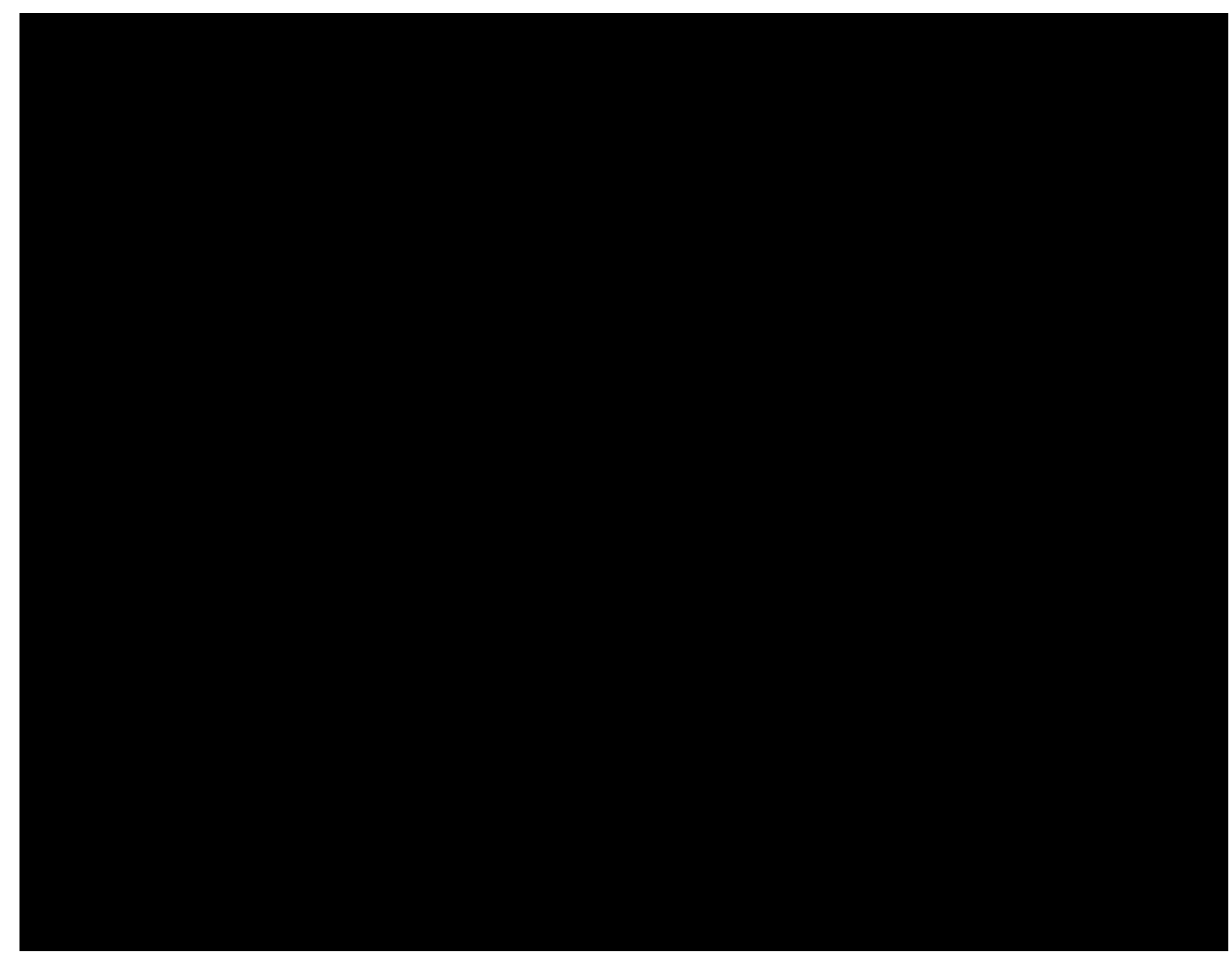

$93 \times 72 \mathrm{~mm}(150 \times 150 \mathrm{DPI})$ 
1

2

3

4

5
6

7

8

10

11

12

13

14

15

16

17

18

19

20

21

22

23

24

25

26

27

28
29

30

31

32

33

34

35

36

37

38

39

40

41

42

43

44

45

46

47

48

49

50

51

52

53

54

55

56

57

58

59

60

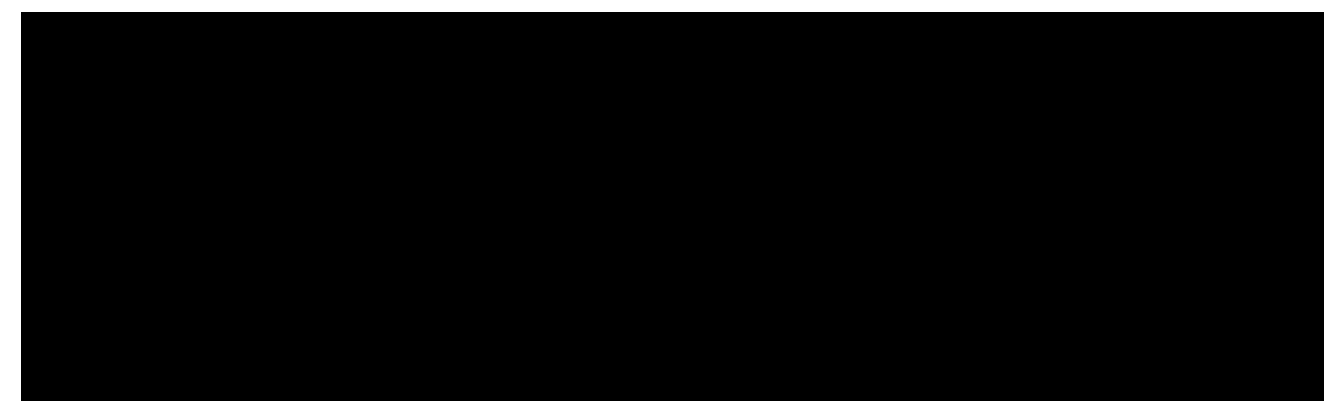

$254 \times 75 \mathrm{~mm}(150 \times 150 \mathrm{DPI})$

Wiley-VCH 


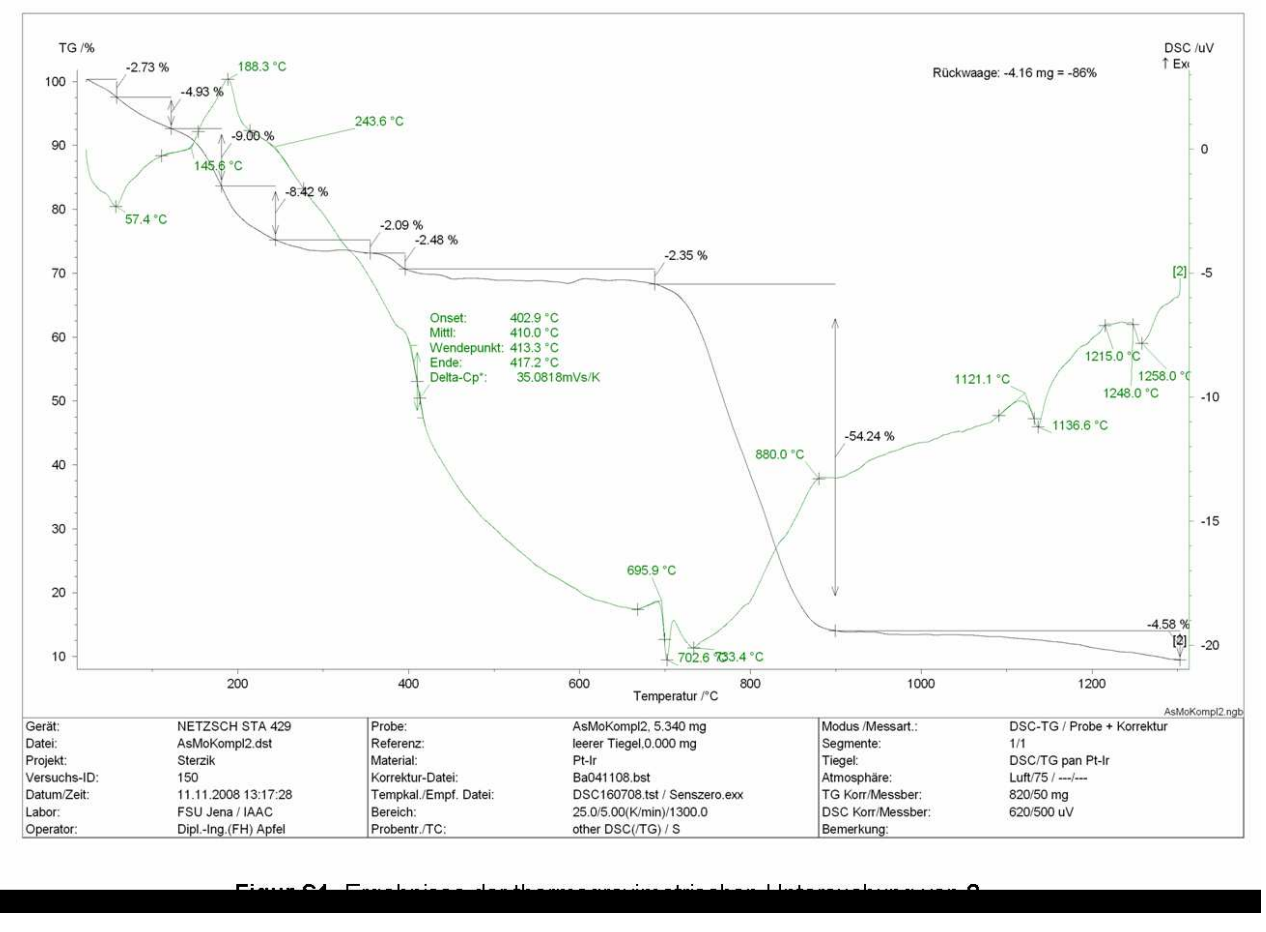

$227 \times 166 \mathrm{~mm}(150 \times 150 \mathrm{DPI})$ 

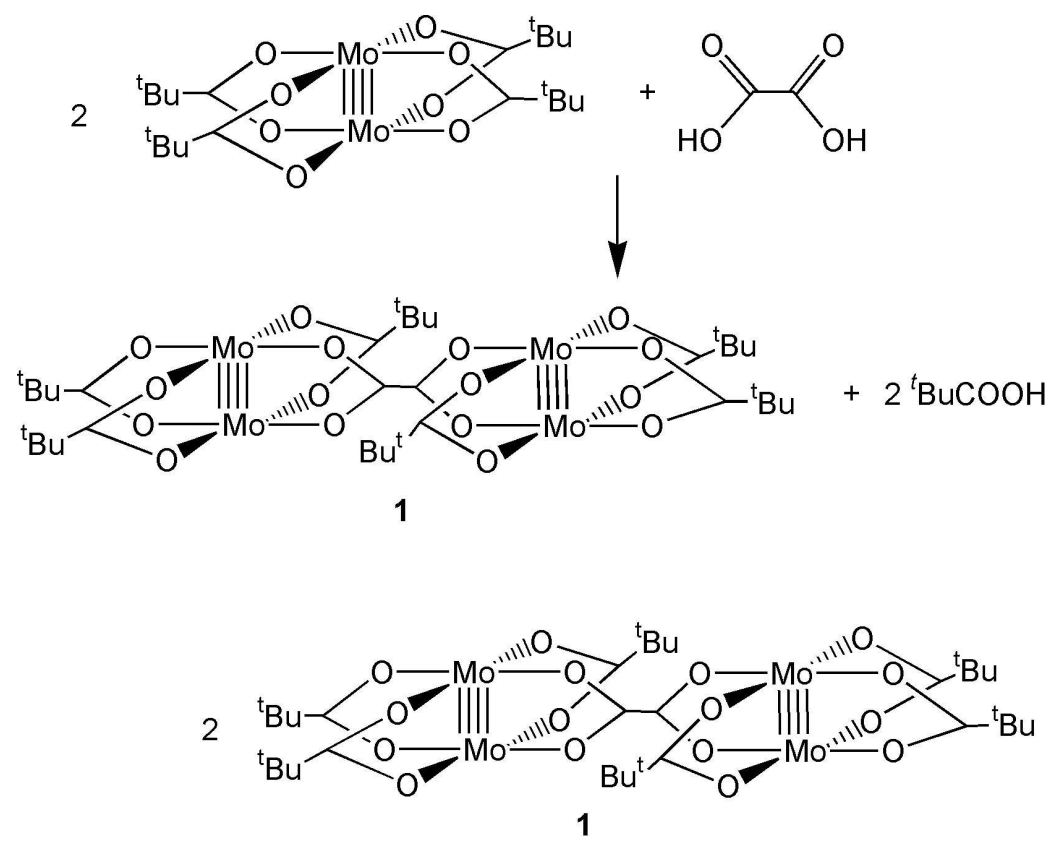

\begin{tabular}{l|l}
$+4 \mathrm{Mnl}_{2}(\mathrm{THF})_{2}$ & $-8{ }^{\mathrm{t}} \mathrm{BuCOOH}$ \\
$+8 \mathrm{CH}_{3} \mathrm{OH}$ & $-3 \mathrm{I}_{2}$ \\
$+11 \mathrm{O}_{2}$ & $-\mathrm{Mn}\left(\mathrm{IO}_{3}\right)_{2}$ \\
& $\left.-2 \mathrm{Mn}^{\mathrm{t}} \mathrm{BuCOO}\right)$ \\
& $-2 \mathrm{CO}_{2}$
\end{tabular}

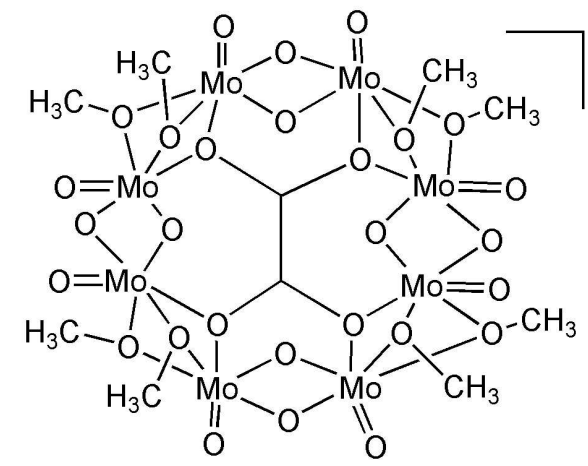<smiles>[H][R](C)(OC)[Y1](O[2H])(OC)(OC)(OC)OC</smiles> 


$$
\begin{aligned}
& 8 \mathrm{Mo}^{2+} \longrightarrow 8 \mathrm{Mo}^{5+}+24 \mathrm{e}^{-} \\
& 8 \mathrm{I}^{-}+3 \mathrm{O}_{2} \longrightarrow 2 \mathrm{IO}_{3}^{-}+3 \mathrm{I}_{2}+6 \mathrm{e}^{-} \\
& \mathrm{C}_{2} \mathrm{O}_{4}{ }^{2-} \\
& 8 \mathrm{O}_{2}+32 \mathrm{e}^{-} \longrightarrow 2 \mathrm{CO}_{2}+2 \mathrm{e}^{-} \\
&
\end{aligned}
$$

